\title{
New Insights into Aging Process with a Focus on Cell Morphology, Autophagy and Role of Macrophages and NFAT
}

\author{
Manoj G Tyagi \\ Department of Biotechnology, Vellore Institute of TechnologyVellore, Tamilnadu, India
}

\begin{abstract}
Aging is a multifaceted process and involves cell senescence and apoptosis. Some key processes which may be involved could be the persistantmorphological changes in the cell structure for e.g cell blebbing and the role of physiological stressors. Amongst these are the macrophages which are on the front line of host defense. They possess an array of germline-encoded pattern recognition receptors/sensors (PRRs) that recognize pathogen-associated molecular patterns (PAMPs) and which activate downstream effectors/pathways to help mediate innate immune responses and host defense. Innate immune responses include the rapid induction of transcriptional networks that trigger the production of cytokines, chemokines, and cytotoxic molecules and the induction of autophagy. Autophagy is a catabolic process that normally maintains cellular homeostasis in a lysosome-dependent manner, but it also functions as a cytoprotective response that intersects with a variety of general stress-response pathways.This review focusses on the intimately linked molecular mechanisms that help govern the autophagic pathway and cyto-morphological changes alongwith macrophage mediated responses to osmolar changes and the role of transcription factors like the NFAT-5 in the aging process.
\end{abstract}

Keywords: Macrophages, autophagy, cell blebbing, NFAT-5, aging

\section{Introduction}

It has been a human endeavour to combat the aging process and increase longevity of life and the the research in this area has now become intensive after the advancement in gene and cell biology techniques and technological breakthroughs. The biological consequences of ageing are similar across mammalian species and include curvature of the spine, impairments in eyesight and hearing, frailty, greying, cognitive impairments, and immune decline [1]. Research in autophagy has been in limelight because of a Nobel Prize awarded to Dr.YoshunoriOhsumi. Amongst the area of interest is the autophagy in cells [2]. Autophagy and cell blebbing may be key processes in aging of cells and tissues. The process of autophagy includes sequestration of longlived proteins and bulky cytosolic contents into double-bilayer vesicular compartments followed by their delivery to lysosomes for degradation [2]. The final metabolites of lysosomal activity are then reused to fulfill energy and new macromolecular needs of the cell. The autophagic process functions as an inherent intracellular recycling mechanism [3]. Autophagic machinery is activated in response to various cellular stressors and often has a cytoprotective function [4]. Depending on the nature of the trigger, either autophagy may proceed as a nonselective bulk degradation process or selectively labeled substrates may be targeted for degradation [5]. Nutrient deprivation, damaged or excessive organelles, accumulated misfolded proteins, endoplasmic reticulum stress, oxidative stress, certain toxins, radiation, and hypoxia can all trigger autophagy [4]. The reactive nature of autophagy gives rise to its participation in a wide array of physiologic and pathologic pathways involved in cell survival, tumor suppression, lifespan extension, cell death, cell differentiation, organismal development, and immunity [5,6]. As a consequence defects in autophagic machinery can cause or contribute to cancer, neurodegenerative diseases, myopathies, immune deficiencies, and premature aging [7]. The hallmark of autophagy is the formation of double-membrane vesicles called autophagosomes. The sources of autophagosome membrane and the factors underlying autophagosome membrane dynamics are complex and a substantial body of literature has addressed their initial formation [8]. Autophagosomes emerge in the cytoplasm as an autophagicat cup shaped protrusions termed omegasomes. These often arise from the endoplasmic reticulum (ER) at sites rich in phosphatidylinositol-3-phosphate (PtdIns3P) and double FYVE-containing protein 1 (DFCP1). On the other hand the macrophages constitute a critical cell type in the innate immune responses [9-10]. They are equipped with germline-encoded pattern recognition receptors/sensors (PRRs) that aid in the recognition of various moieties from microbes termed pathogen-associated molecular patterns (PAMPs) and also danger-associated molecular patterns (DAMPs) [11]. Lipids, nucleic acids, proteins, lipoproteins, glycans derived from a range of bacteria, viruses, parasites, and fungi are designated as PAMPs. Depending on the specific receptor-PAMP/DAMP match and whether multiple PRRs are engaged, various downstream effectors/pathways are activated, which prepare the cell to combat the invading agents by activating 
degradation pathways and relaying signals such as cytokines to alert other cells of the innate and adaptive immune system in the surrounding tissues and at distal sites [12].Disturbance in autophagy can cause cell blebbing and lead to deleterious effects on cell life. Correlation of intracellular $\mathrm{Na}^{+}$loading with bleb initiation strongly implicates $\mathrm{Na}^{+}$as a trigger.Na ${ }^{+}$itself has been shown to stimulate vesicular machinery by altering cAMP levels, leading to activation of cAMP-dependent protein kinase (PKA) [12]. This could be the basis of our ability to block bleb formation with ATP depletion, cAMP inhibition, or inhibition of PtdIns 3-kinase. Autophagy induction in response to these signals may promote the removal of damaged proteins or organelles and maintain proper metabolism, which is crucial for cell survival.

Mitochondria play an important role in aging and regulate autophagy through ROS generation and the MPT . Autophagy induction signaled from mitochondria can result in enhanced survival or may possibly contribute to cell death [13-14].

\section{Cell blebbing and autophagy:}

Cell blebs are spherical cellular membrane protrusions that inflate and retract on a timescale of minutes, resulting from either the detachment of the cell membrane from the actin cortex [15] or the localized rupture of the actin cortex . Cell blebs attract a great deal of interest because of their dynamic features connected to dramatic cellular reorganization with the roles in cytokinesis, cell spreading [16].Blebbing may not be the only factor that can protect cells because the hypertonic stress may cause autophagy and thus improve the cell survival [17]. The hypertonic stress results in the breakage of the cortex, causing blebbing and autophagy, which can both enhance cell survival. However, the corresponding mechanisms of cell protection are different in the two cases: blebbing directly releases the stress, whereas autophagy indirectly inhibits the apoptosis. The first mechanism is faster than the second process.Plasma membrane blebs are usually defined as hemispherical protrusions at the cell surface. Blebs are found in association with cell injury and subsequent cellular necrosis or apoptosis [18]. Blebs also have been observed in physiologically healthy cells at particular stages of the cell cycle [19]. As an example, when mesoderm cells migrate within the embryo, prominent blebs can be observed in situ. In addition, neurons differentiating in primary culture show circus movements, i.e., blebs circulating around the cell circumference [20]. The existence of autophagic cell death remains a topic for debate because autophagy is also a mechanism to maintain homeostasis. Controlled breakdown of intracellular components provides a nutrient supply during starvation and other stresses where removal of damaged organelles, such as mitochondria with reduced membrane potential, can protect cells from damage. The mechanism of how autophagy contributes to cell death is largely unknown. Both apoptotic and autophagic processes may be regulated by the same pathways, although one form of cell death can be enhanced when the other is blocked. As such, activation of autophagy duringdeath may be a cause of lethality or may actually be a futileattempt at rescue. The DAPK-1 family proteins are one ofthe first molecules described to directly regulate autophagyas a mode of cell death. It has been proposed that autophagiccell death is a more ancient-based type of cell death program,because many of the autophagy-associated genes are evolutionarily conserved between yeast and mammalian organisms. The DAPK-1 family proteins are one ofthe first molecules described to directly regulate autophagyas a mode of cell death. It has been proposed that autophagiccell death is a more ancient-based type of cell death program,because many of the autophagy-associated genes are evolutionarily conserved between yeast and mammalian organisms. The upregulation of autophagy in long-lived mutants would likely enable the cell to endure stressful conditions by increasing the rate of turnover of damaged macromolecules. It is possible that autophagy promotes cell maintenance by removing accumulated toxic material and by using recycled components as an alternative nutrient resource. This suggests that autophagy favors longevity because an organism can recover more quickly from stress-induced cellular damage. Evidence that autophagy influences the aging process has been observed in multiple model organisms, from yeast to multicellular organisms such as worms and flies, and a more recent and exciting finding is that autophagy is implicated in neurodegenerative diseases that affect humans. A defect in the autophagy and cell blebbing does have a impact on cell senescence and over aging of tissues in higher mammals including humans.

\section{Technique of apoptosis induction and bleb isolation:}

Apoptosis is induced with bile salts as described previously [21-22], with minor modifications. Briefly, PKH-26-labelled or unlabelledHiBECs were incubated in culture medium containing $1 \mathrm{mM}$ sodium glycochenodeoxycholate (GCDC; Sigma-Aldrich) at $37^{\circ} \mathrm{C}$ for $4 \mathrm{~h}$. The apoptosis rate was assessed by flow cytometry after staining with 7-aminoactinomycin D (7-AAD) and fluorescein isothiocyanate (FITC)-annexin V (BD Biosciences, San Jose, CA, USA). More than 70\% of the cells undergo early apoptosis (annexin V-positive and 7-AAD-negative) after treatment. To isolate blebs from apoptotic HiBECs, the culture supernatants were collected and centrifuged twice at $500 \mathrm{~g}$ for $5 \mathrm{~min}$ to remove the remaining cells. The supernatants were then centrifuged at $100000 \mathrm{~g}$ for $45 \mathrm{~min}$ at $4^{\circ} \mathrm{C}$. The pellets containing blebs were resuspended in EpiCM. The bleb 
numbers were counted as we have described previously [23]. For obtaining apoptotic thymocytes, PKH-26stained thymocytes were irradiated at $30 \mathrm{~Gy}$ (Caesium-137 irradiator, $627 \mathrm{R} / \mathrm{min}$ ), washed and analysed using the fluorescence-based apoptosis assay.

\section{47 and cell blebbing in macrophages:}

As a marker protein for self-recognition, CD47 plays a critical role in protecting host cells/tissues from being attacked by host phagocytes. Accumulating evidence has shown that ligation of macrophage SIRP $\alpha$ by CD47 expressed on the encountered cells prohibits macrophage phagocytosis, whereas failure of SIRP $\alpha$ engagement by CD47, or deficiency of SIRP $\alpha$ ITIM-mediated signaling, promotes macrophage engulfment of the host cell [24-26] has been reported that cells likely lose their surface CD47 when aging or undergoing apoptosis, and this change results in abrogation of the CD47-SIRP $\alpha$-mediated inhibitory signaling and hence facilitates macrophage phagocytic clearance. Along these dramatic cellular changes, CD47 at the stage of apoptosis shows remaining on the cell body without distribution to blebs, in a way very similar to the pattern of actin as reported by Charraset al [27], suggesting that CD47 may through its cytoplasmic domain connect to the underlying cytoskeleton cortex. Not only are they CD47-negative, the blebs at the stage of apoptosis are small and dynamic. In contrast, at the stage of necrosis membrane blebs are large and tend to detach from the cell body, and these blebs are CD47-positive. Therefore, the decrease in CD47 on necrotic cells could be the result of shrinkage of the cell size and loss of the plasma membrane, even though CD47 molecules per membrane area may not reduce. Results showed that cell apoptosis does not decrease the CD47 level on the cell surface but alters the cell surface pattern of CD47 from 'punctate' clusters into diffused distribution, which dramatically decreases the avidity of CD47-mediated cell binding to SIRP $\alpha$ and incapacitates SIRP $\alpha$-mediated inhibitory signalling in macrophages [28-30].

\section{Macrophage polarization and differentiation in aging process:}

It is important to note that macrophages are extremely heterogeneous. Tissue-resident macrophages are distributed widely throughout the body and functional capabilities and morphology varies between sites [31-33]. For example, Kupffer cells in the liver are involved in the clearance of pathogens and toxins from the circulation. Alveolar macrophages in the lungs eliminate dust, allergens, and microorganisms from the airway, and osteoclasts in the skeletal system play a role in bone resorption.In a mouse model of age-related macular degeneration, macrophages from old mice polarised towards a proangiogenic phenotype. IL-10 was upregulated, and IL-12 and TNF- $\alpha$ were downregulated in ocular macrophages in old mice; such macrophages were unable to inhibit angiogenesis following laser injury to the retina [34]. Interestingly, it has also been reported that there is an increase in M2 macrophages in spleen, lymph nodes, and bone marrow of old mice [35]. It is unclear whether generation and differentiation of macrophages from precursors are impaired with age. A decrease in $\mathrm{CD} 68^{+}$cells in bone marrow with age has been observed in humans. In contrast, it has been reported that $\mathrm{Mac}^{+}$cells are increased in bone marrow in old mice. Others have reported no difference in number, size, or Mac1 expression during macrophage maturation in old mice [36]. Further investigation of the impact of ageing on macrophage differentiation is needed in light of the recent advances in understanding of macrophage differentiation.

\section{A nexus between NFAT5 and macrophage function and development implications for aging:}

Macrophage survival under hyperosmolar conditions may have connection to the NFAT 5 and could be key to the survival of cells in normal physiological and pathological conditions.Hyperosmolality may improve the life span of macrophages and thereby affect apoptosis of cells. In this connection the transcription factor p53 is critical and several signaling pathways that are essential for cell-growth regulation and apoptosis [37-38]. In normal conditions, the level of p53 in the cells is usually limited by a constant degradation of the protein by the ubiquitin/proteasome pathway and play an important role in several cell types [39]. When normal mouse macrophages were put in culture in isosmotic conditions, it was found that the p53 level is slightly increased until cell death. Consistently, in isosmotic cultures, Bcl-2, a pro-survival protein is down-regulated while Bax, a pro-apoptotic gene transactivated by p53, is overexpressed [40]. However, hyperosmolarity prevents p53 and Bax regulation as well as $\mathrm{Bcl}-2$ inhibition. This suggests that hyperosmolarity prevents apoptosis and allows macrophage half-life to be extended. These might be the initial events in a cell transformation process. During inflammation, there is normally an influx of macrophages and a high rate of epithelial cell death can occur. Recent data strongly suggests that hyperosmolarity appears to play a key role both in the recruitment and the survival of macrophages.

\section{Conclusion}

One of the enigma of aging research is of course to find a fountain of youth an elixir that will prolong life. Although several commercial entities are unleashing their resources at such a goal, given the inconclusive nature of most molecular aging pathways currently under consideration, these efforts seem unlikely to come to fruition in the near future. However, therapeutic intervention may be realistic for premature aging disorders as 
their molecular basis is becoming well defined. The feasibility of anti-aging intervention is made clearer and the pursuit of the biological basis of aging has been revitalized within the last fifteen years by studies in yeast, worms, flies, and mice that have firmly established that there indeed exist specific molecular mechanisms that contribute to the aging process. These efforts point to several distinct, likely interrelated, mechanisms, ranging from imbalance in protein metabolism, to alterations of specific signalling pathways, progressive damage due to generation of oxidative free radicals, and increased genomic instability. This review article has given newer insights into the process of aging by focusing on the role of cell blebbing and autophagy and the link with macrophages and NFAT 5 in their contribution to the physiological aging process and possibility of avenues to reverse this trend in the future.

Acknowledgement: The author wishes to thank the Department of Molecular Pharmacology, University of Nottingham, United Kingdom.

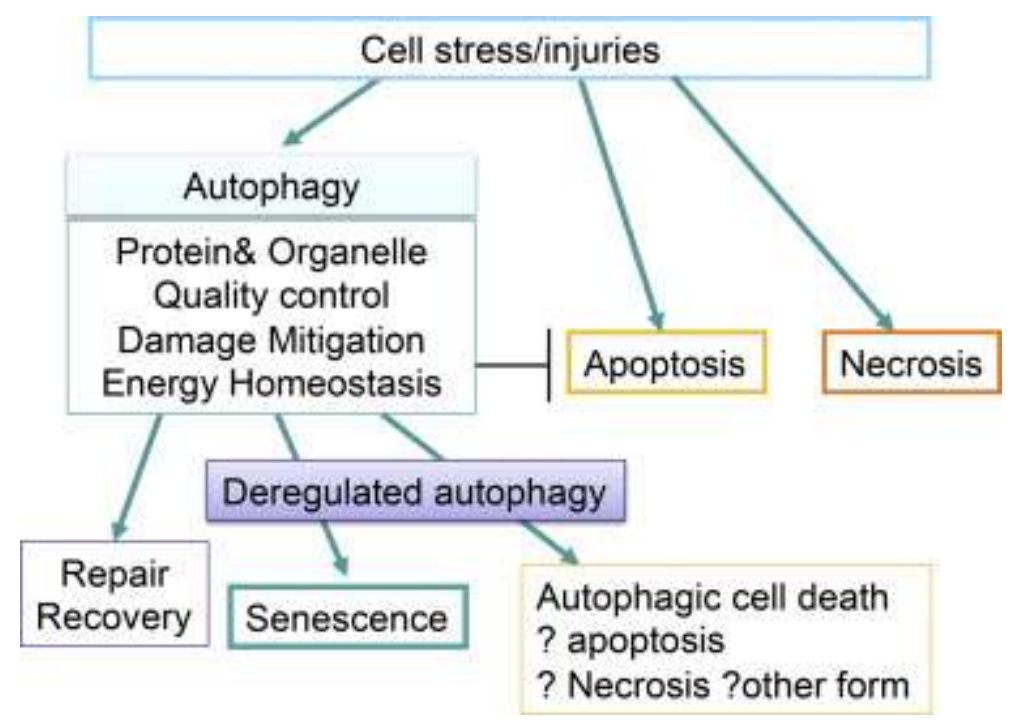

Fig.1: Autophagy and cell death and aging

\section{References}

[1]. B. Levine, N. Mizushima, and H. W. Virgin, “Autophagy in immunity and inflammation,” Nature, vol. 469, no. 7330, pp. 323-335, 2011.

[2]. Keisuke Mochida, Yu Oikawa, Yayoi Kimura, Hiromi Kirisako, Hisashi Hirano, Yoshinori Ohsumi\& Hitoshi Nakatogawa.Receptor-mediated selective autophagy degradesthe endoplasmic reticulum and the nucleus.Nature, 2015, 522 ,359374

[3]. P. Boya, F. Reggiori, and P. Codogno, "Emerging regulation and functions of autophagy," Nature Cell Biology, vol. 15, no. 7, pp. 713-720, 2013.

[4]. C. C. Mihalache and H.-U. Simon, "Autophagy regulation in macrophages and neutrophils," Experimental Cell Research, vol. 318, no. 11, pp. 1187-1192, 2012.

[5]. Z. Yang and D. J. Klionsky, "Mammalian autophagy: core molecular machinery and signaling regulation," Current Opinion in Cell Biology, vol. 22, no. 2, pp. 124-131, 2010.

[6]. N. Mizushima, B. Levine, A. M. Cuervo, and D. J. Klionsky, “Autophagy fights disease through cellular self-digestion,” Nature, vol. 451, no. 7182, pp. 1069-1075, 2008 .

[7]. P. J. Murray and T. A. Wynn, "Protective and pathogenic functions of macrophage subsets," Nature Reviews Immunology, vol. 11, no. 11 , pp. 723-737, 2011.

[8]. V. Deretic, T. Saitoh, and S. Akira, "Autophagy in infection, inflammation and immunity," Nature Reviews Immunology, vol. 13, no. 10 , pp. $722-737,2013$.

[9]. L. Gong, R. J. Devenish, and M. Prescott, “Autophagy as a macrophage response to bacterial infection,” IUBMB Life, vol. 64, no. 9, pp. 740-747, 2012

[10]. J. D. Mintern and J. A. Villadangos, "Autophagy and mechanisms of effective immunity," Frontiers in Immunology, vol. 3, article 60, 2012.

[11]. Nunes P, Ernandez T, Roth I, Qiao XM, Strebel D, Bouley R, et al. Hypertonic stress promotes autophagy and microtubuledependent autophagosomal clusters. Autophagy. 2013;9(4):550-67.

[12]. Klionsky DJ, Emr SD. Autophagy as a regulated pathway of cellular degradation. Science 290: 1717-1721, 2000

[13]. Rajawat YS, Bossis I (2008) Autophagy in aging and in neurodegenerative disorders. Hormones (Athens) 7: 46-61.

[14]. Paluch E, Piel M, Prost J, Bornens M, Sykes C. Cortical actomyosin breakage triggers shape oscillations in cells and cell fragments. Biophys J. 2005;89(1):72, 4-33. 
[15]. Charras G, Paluch E. Blebs lead the way: how to migrate without lamellipodia. Nature reviews Molecular cell biology. 2008;9(9):730-6

[16]. RenquanRuan, Lili Zou, Sijie Sun, Jing Liu, Longping Wen, Dayong Gao, Weiping Ding Cell Blebbing upon Addition of Cryoprotectants: A Self-Protection Mechanism. Plos one, April 13, 2015

[17]. Keller HU and Bebie H. Protrusive activity quantitatively determines therate and direction of cell locomotion.CellMotil Cytoskeleton33: 241-251, 1996

[18]. Alfieri RR, Petronini PG.Hyperosmotic stress response: comparison with other cellular stresses. PflugersArchiv: European journal of physiology. 2007;454(2):173-85

[19]. Norman LL, Brugues J, Sengupta K, Sens P, Aranda-Espinoza H. Cell blebbing and membrane area homeostasis in spreading and retracting cells. Biophys J. 2010;99(6):1726-33.

[20]. A. Chow, B. D. Brown, and M. Merad, "Studying the mononuclear phagocyte system in the molecular age," Nature Reviews Immunology, vol. 11, no. 11, pp. 788-798, 2011.

[21]. Lleo A, Selmi C, Invernizzi P, et al. Apotopes and the biliary specificity of primary biliary cirrhosis. Hepatology. 2009;49:871-879.

[22]. Rong G, Zhong R, Lleo A, et al. Epithelial cell specificity and apotope recognition by serum autoantibodies in primary biliary cirrhosis. Hepatology. 2011;54:196-203

[23]. Lleo A, Bowlus CL, Yang G-X, et al. Biliary apotopes and anti-mitochondrial antibodies activate innate immune responses in primary biliary cirrhosis. Hepatology. 2010;52:987-998.

[24]. Matozaki T, Murata Y, Okazawa H, Ohnishi H. Functions and molecular mechanisms of the CD47-SIRP $\alpha$ lphasignalling pathway. Trends Cell Biol. 2009;19:72-80.

[25]. Barclay AN, Van den Berg TK. The interaction between signal regulatory protein alpha (SIRP $\alpha$ lpha) and CD47: structure, function, and therapeutic target. Annu Rev Immunol. 2014;32:25-50.

[26]. Takizawa H, Manz MG. Macrophage tolerance: CD47-SIRP-alpha-mediated signals matter. Nat Immunol. 2007;8:1287-1289.

[27]. Ide K, Wang H, Tahara H, Liu J, Wang X, Asahara T, Sykes M, Yang YG, Ohdan H. Role for CD47-SIRP $\alpha$ lpha signaling in xenograft rejection by macrophages. Proc Natl AcadSci U S A. 2007;104:5062-5066.

[28]. Oldenborg PA, Zheleznyak A, Fang YF, Lagenaur CF, Gresham HD, Lindberg FP. Role of CD47 as a marker of self on red blood cells. Science. 2000;288:2051-2054.

[29]. Ogawa T, Kitagawa M, Hirokawa K. Age-related changes of human bone marrow: a histometric estimation of proliferative cells, apoptotic cells, T cells, B cells and macrophages. Mech Ageing Dev. 2000;117:57-68.

[30]. Kelly J, Ali Khan A, Yin J, Ferguson TA, Apte RS. Senescence regulates macrophage activation and angiogenic fate at sites of tissue injury in mice. J Clin Invest. 2007;117:3421-3426.

[31]. Wang CQ, Udupa KB, Xiao H, Lipschitz DA. Effect of age on marrow macrophage number and function. Aging (Milano) 1995;7:379-384.

[32]. Ikeda H, Okazawa H, Ohnishi H, Murata Y, Oldenborg PA, Matozaki T. Mutational analysis of the mechanism of negative regulation by SRC homology 2 domain-containing protein tyrosine phosphatase substrate-1 of phagocytosis in macrophages. $\mathrm{J}$ Immunol. 2006;177:3123-3132.

[33]. Jackaman C, Radley-Crabb HG, Soffe Z, Shavlakadze T, Grounds MD, Nelson DJ. Targeting macrophages rescues age-related immune deficiencies in C57BL/6J geriatric mice. Aging Cell. 2013;12:345-357.

[34]. Wynn TA, Chawla A, Pollard JW. Macrophage biology in development, homeostasis and disease. Nature. 2013;496:445-455.

[35]. Lee CK, Weindruch R, Prolla TA. Gene-expression profile of the ageing brain in mice. Nat Genet25: 294-297, 2000.

[36]. Choi S, You S, Kim D, Choi SY, Kwon HM, Kim HS, Hwang D, Park YJ, Cho CS, Kim WU.Transcription factor NFAT5 promotes macrophage survival in rheumatoid arthritis.J Clin Invest. 2017 Mar 1;127(3):954-969.

[37]. N Sawhney, MK Patel, M Schachter and AD Hughes. Inhibition of proliferation by heparin and expression of p53 in cultured human vascular smooth muscle cells. Journal of Human Hypertension (1997) 11, 611-614

[38]. Labi V, Erlacher M, Kiessling S, Villunger A: BH3-only proteins in cell death initiation, malignant disease and anticancer therapy. Cell Death Differ. 2006, 13:1325-1338

[39]. Prattichizzo F, Bonafè M, Olivieri F, Franceschi C Senescence associated macrophages and "macro-phaging": are they pieces of the same puzzle? Aging (Albany NY). 2016 Dec 7;8(12):3159-3160

[40]. Manoj G Tyagi.TonEBP and its Role in Physiological Regulation and Aging Process. Research Journal of Pharmaceutical, Biological and Chemical Sciences. August 2014, RJPBCS,5(4), 450-456 